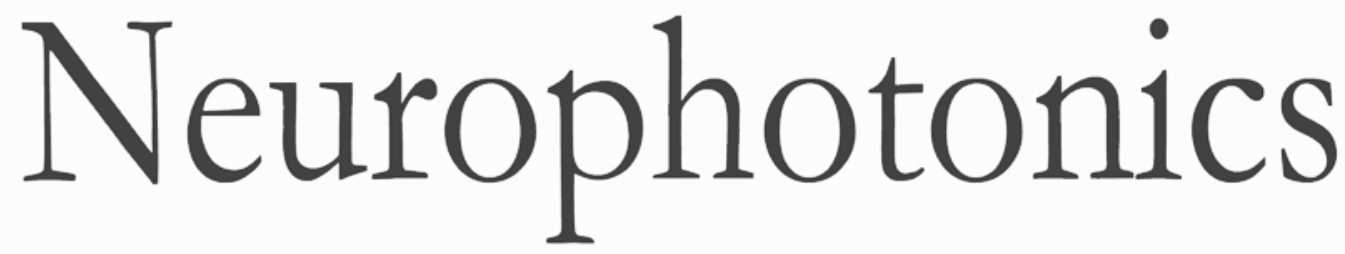

\title{
Visual stimulation quenches global alpha range activity in awake primate V4: a case study
}

Thomas Deneux

Timothée Masquelier

Maria A. Bermudez

Guillaume S. Masson

Gustavo Deco

Ivo Vanzetta 


\title{
Visual stimulation quenches global alpha range activity in awake primate V4: a case study
}

\author{
Thomas Deneux, ${ }^{\mathrm{a}, \mathrm{b}}$ Timothée Masquelier, ${ }^{\mathrm{c}, \mathrm{d}}$ Maria A. Bermudez, ${ }^{\mathrm{a}}$ Guillaume S. Masson, ${ }^{\mathrm{a}}$ Gustavo Deco, ${ }^{\mathrm{c}}$ and \\ Ivo Vanzetta ${ }^{a, \star}$ \\ alnstitut de Neurosciences de la Timone, UMR 7289, CNRS and Aix-Marseille Université, Marseille, France \\ bUnit of Neuroscience Information and Complexity, CNRS, Gif-sur-Yvette, France \\ 'Universitat Pompeu Fabra, Department of Technology, Barcelona, Spain \\ ${ }^{\mathrm{d}}$ Institut de la Vision (CNRS-UPMC), Centre de Recherche Cerveau et Cognition (CNRS-UT3), Toulouse, France
}

\begin{abstract}
Increasing evidence suggests that sensory stimulation not only changes the level of cortical activity with respect to baseline but also its structure. Despite having been reported in a multitude of conditions and preparations (for instance, as a quenching of intertrial variability, Churchland et al., 2010), such changes remain relatively poorly characterized. Here, we used optical imaging of voltage-sensitive dyes to explore, in V4 of an awake macaque, the spatiotemporal characteristics of both visually evoked and spontaneously ongoing neuronal activity and their difference. With respect to the spontaneous case, we detected a reduction in large-scale activity (cortical extent $>1 \mathrm{~mm}$ ) in the alpha range $(5$ to $12.5 \mathrm{~Hz}$ ) during sensory inflow accompanied by a decrease in pairwise correlations. Moreover, the spatial patterns of correlation obtained during the different visual stimuli were on the average more similar one to another than they were to that obtained in the absence of stimulation. Finally, these observed changes in activity dynamics approached saturation already at very low stimulus contrasts, unlike the progressive, near-linear increase of the mean raw evoked responses over a wide range of contrast values, which could indicate a specific switching in the presence of a sensory inflow. (C) 2017 Society of Photo-Optical Instrumentation Engineers (SPIE) [DOI: 10.1117/1.NPh.4.3.031222]
\end{abstract}

Keywords: V4; awake monkey; optical imaging; voltage-sensitive dyes; ongoing activity; correlation.

Paper 17009SSR received Jan. 15, 2017; accepted for publication Jun. 8, 2017; published online Jun. $28,2017$.

\section{Introduction}

Over the last 20 years, the view on spontaneously ongoing activity has changed from essentially random noise-to be eventually discarded as a nuisance to study stimulus-dependent response features - to a nonrandom network activity of the same order of magnitude as that evoked by a sensory input ${ }^{1}$ and interacting with the latter. ${ }^{2-6}$ In particular, it has been reported that the spatiotemporal structures of such activity as measured with optical imaging of voltage-sensitive dyes (VSDs) share similarities with evoked responses, ${ }^{7}$ and it has thus been suggested that spontaneous activity reflects or influences memory, perception, and behavior. Moreover, chronic multielectrode recordings have recently provided strong evidence that ongoing activity is shaped during development by the sensory inputs and that in their absence, it displays dynamics that are characteristic of the most common inputs that a given area has processed in the past. ${ }^{8}$

The literature also abundantly documents changes in the statistics of neuronal activity during or following stimulation. For instance, a reduction in the variability of neuronal activity across trials has been reported, both in the form of spiking activity and membrane potential changes ${ }^{9-14}$ and in the form of hemodynamics measured by blood oxygenation level dependent functional magnetic resonance imaging. ${ }^{15} \mathrm{~A}$ recent study ${ }^{16}$ has systematically investigated these changes in response variability in primary visual cortex, in the across-trial sense as well as in the sense of the temporal fluctuations of the single-trial response

*Address all correspondence to: Ivo Vanzetta, E-mail: ivo.vanzetta@univ-amu with respect to the trial mean (our "noise signal," see below), and modeled them within a sampling-based probabilistic representation framework. An early transient drop in the correlated variability of the spiking of neuronal pairs has also been reported. ${ }^{17,18}$ Finally, in anesthetized rat barrel cortex, brief sensory stimulation has been shown to induce a decrease in ongoing activity lasting several seconds, ${ }^{19}$ i.e., far beyond the end of the sensory inflow itself.

Despite this multitude of experimental studies, however, a detailed spatiotemporal characterization of the sensory-induced modifications in the structure of neuronal activity-its statistics and its variability in particular-is still missing, especially in the awake primate.

Here, we provide such information in the form of neuronal population data obtained in extrastriate area V4 of an awake macaque monkey, during the presentation of full-field drifting isoluminant or luminance-based gratings and during the presentation of a uniformly gray screen. As a recording technique, we used optical imaging of VSDs because in typical VSD imaging settings in vivo, each pixel averages the signals from 150 to 200 neurons, ${ }^{20}$ allowing to overcome - to a great extent at least- the sampling issues affecting microelectrode recordings (including multielectrode arrays). In addition, the excellent spatial resolution of VSD imaging $(\sim 30 \mu \mathrm{m})$, its large field of view, and its high temporal resolution (here over $10 \mathrm{~mm}^{2}$ and $10 \mathrm{~ms} /$ frame, respectively) provide access to a substantial part of the cortical dynamics in the area of interest. As a result, we could characterize, at the subcolumnar resolution, the full spatiotemporal

2329-423X/2017/\$25.00 @ 2017 SPIE 
dynamics of neuronal population activity during the whole recorded trial (here nearly $1 \mathrm{~s}$ ), over an area the size of several cortical columns.

Our first finding is a decrease of global ( $>1 \mathrm{~mm})$ activity in the alpha frequency range following sensory stimulation, both in the raw signals and in what we call the "noise signal," i.e., the deviation, at the single trial level, of the signal from the trial average.

Second, we report a sensory-induced reduction in pairwise correlation of this noise signal (for details and a comparison with "classical" variability and correlations, see Appendix A). In addition to and beyond a similar correlation drop reported previously, ${ }^{21}$ here we provide a detailed characterization of these changes in correlation in the spatial, temporal, and, in particular, the spectral domain.

Our last finding concerns the peculiar contrast dynamics of some of these sensory-induced changes in the spatiotemporal structure of cortical activity, which was already saturated for visual stimuli of very low contrasts, in a range where the raw response amplitudes still grew near-linearly. Together with our data on the spectral structure of sensory-induced changes in cortical dynamics, this last result might provide experimental testing ground to recent modeling efforts ${ }^{18}$ aimed to explain cortical dynamics in V1, which, as opposed to most other studies, investigated not only intertrial variability but also the variability of noise within trials, its pairwise correlation between cells, and the dependence of those variables on stimulus contrast.

\section{Methods}

\subsection{Preparation}

Experiments were performed on one adult male monkey (Macaca mulatta), with a transparent cranial window chronically implanted onto a $1.8-\mathrm{cm}$ diameter aperture in the skull above visual area V4, comprehending $\sim 2.5 \mathrm{deg}$ to $15 \mathrm{deg}$ eccentricity of the visual field. For a detailed description of all surgical and maintenance procedures, see Refs. 22 and 23 and the references therein. Before imaging, the cortex was stained for $3 \mathrm{~h}$ via superfusion with RH 1691 or RG 1883 (for details, see Ref. 22). All procedures were approved by the local ethical committee and were in accordance with European guidelines.

\subsection{Experimental Paradigm}

The paradigm in the alert monkey experiments was a simple fixation task. A trial started when the monkey began fixation on a fixation point (size: $0.1 \mathrm{deg}$ to $0.15 \mathrm{deg}$ ), displayed on an otherwise uniformly gray cathode ray tube (CRT) screen [of color and luminance coordinates $(x, y, Y)_{\mathrm{CIE} 1931}=(0.284$, $0.322,43.7)$ in CIE1931 color space]. After $100 \mathrm{~ms}$, a drifting sinusoidal grating appeared for $0.6 \mathrm{~s}$, except in the "blank" conditions (no grating). The stimulus was then turned off, and the monkey had to continue to fixate until the fixation point disappeared, for a total fixation period of $1 \mathrm{~s}$ (including for blank trials). In the present study (and as opposed to other projects performed some months later), the monkey did not have to report on any aspect of the stimulus, such as orientation, direction of motion, or the like. Data from trials where the monkey broke fixation were rejected. To allow for the relaxation of all hemodynamic signals, an intertrial interval of $\sim 10 \mathrm{~s}$ followed during which the CRT screen was uniform gray. All gratings were centered on the same white point $\left[(x, y, Y)_{\mathrm{CIE} 1931}=\right.$ $(0.284,0.322,43.7)]$, and so were the interstimulus and intertrial stimulus, which consisted of a uniform gray screen (identical to the blank, but without the fixation dot).

The stimuli were binocularly presented horizontal and vertical sinusoidal gratings, having a spatial frequency of $0.2 \mathrm{cyc} / \mathrm{deg}$ and drifting at $16 \mathrm{deg} / \mathrm{s}$ in one of the four cardinal directions (amounting to a temporal frequency of $3.2 \mathrm{~Hz}$ ). The gratings could be isoluminant (in the sense of the mean human, the "standard CIE1931 observer"), i.e., based on color (redgreen) or on luminance contrast (dark and bright gray). The stimuli in the two categories were matched for root mean square (RMS) cone contrast: $\mathrm{CC}_{\mathrm{RMS}}=\operatorname{sqrt}\left\{1 / 2\left[(\mathrm{DL} / \mathrm{L})^{2}+(\mathrm{DM} / \mathrm{M})^{2}\right]\right\}$, where $L$ and $M$ stand for the average activity of long and medium wavelength cones, respectively, and DL, DM, and DS denote the amplitude of the $L$ and $M$ cone activity modulation by the drifting grating with respect to the average. ${ }^{24}$ The gratings were presented at six different contrast levels (5\%, $10 \%, 20 \%, 40 \%, 80 \%$, and $100 \%$ ), where $100 \%$ corresponds to the maximal modulation in the red-green direction the CRT monitor could deliver: $\mathrm{CC}_{\mathrm{RMS}}$ (red versus green $)=0.182$. This means that, expressed in $\mathrm{CC}_{\mathrm{RMS}}$ contrasts units, our stimuli had RMS cone contrast values of $0.0091,0.0182,0.0364,0.0728$, 0.1456 , and 0.182 .

\subsection{Optical Recordings}

Data were acquired in three sessions at a distance of a few weeks one from another. Optical recordings were performed at a framerate of $110 \mathrm{~Hz}$, with a commercial imaging system (Imager 3001, Germantown, Maryland), adapted for the behaving monkey. ${ }^{23}$ The exposed cortex was illuminated (100 to $150 \mathrm{~W}$ tungsten halogen lamp, Zeiss, Germany) through a 20-nm bandpass interference filter centered at $630 \mathrm{~nm}$ by a dichroic mirror with a cutoff at $650 \mathrm{~nm}$ (respectively, 630DF20 and 650DRLP, Omega Optical, Brattleboro, Vermont). Fluorescence was collected through the same dichroic mirror and an additional red-pass filter, having a cutoff at $665 \mathrm{~nm}$ (RG 665, 3-mm thick, Schott, Germany). Data were acquired in recording sessions of $\sim 3 \mathrm{~h}$.

\subsection{Data Preprocessing}

Raw data were initially corrected for bleaching and heartbeat artifacts. Bleaching artifact was removed simply by fitting and removing two exponentials from the time courses of each pixel and every trial (the time constants of these exponentials were first estimated on the average blank signal over all trials and pixels, and only their amplitudes were fit to every individual trial and pixel).

Heartbeat artifact removal was particularly delicate, as heartbeat-related signals at 2 to $3 \mathrm{~Hz}$ had similar frequency content with the evoked responses. First, the phase of the heartbeat cycle was estimated in every trial using a Hilbert transform at around $2.5 \mathrm{~Hz}$ performed on signals averaged from a region of interest, where heart contamination was strong and from which the average responses to the stimuli were subtracted. Second, a general linear model estimation was performed on all blank trials (from the same session) to estimate for every pixel a canonical contamination signal during one heart cycle - this signal was assumed to change smoothly from a first template at the beginning of the session to a second template at the end of the session. These two estimations, once combined, allowed reconstituting the heart artifact in every trial and pixel and subtracting it. 
Two additional preprocessings were performed on these corrected signals: signals were divided by average responses to the different stimuli so as to keep only fluctuations that constitute the intertrial variability, and a low-pass filter at cutoff frequency of $25 \mathrm{~Hz}$ (using a Gaussian mask in the Fourier space) removed high-frequency content that mostly contained photonic and camera noises. The text refers to the data after bleaching and heartbeat correction as "raw signals" and after the additional normalization and filtering as "normalized signals." Importantly, no filtering was used before the spectral analysis (Fig. 4).

\subsection{Data Analysis}

In general, analyses were performed on signals restricted to specific time windows (the "onset period" 0 to $250 \mathrm{~ms}$ after stimulation onset and the "plateau period" 250 to $700 \mathrm{~ms}$ after stimulation onset). Before computing power, correlations or applying Fourier transform in these time windows, the signals were systematically detrended to prevent edge effects as much as possible.

Signal power [Fig. 2(a)] was computed individually in every pixel, then averaged over pixels and trials of the same conditions. Signal correlations [Fig. 2(b)] were computed first for every pixel pair, then averaged over pixel pairs and over trials. For computing these quantities over all three sessions together [top panels in Figs. 2(a)-2(b)], trial averaging was performed on all trials from the three sessions pooled together.

For the grouping of pixels according to distances for the spatial analysis [Fig. 3(b)] and the correction of correlations by an estimated "distance effect" [Figs. 3(c) and 3(d)], we proceeded as follows:

First, we grouped pixel pairs into six bins according to the distance between the members of a pair (150 to $300 \mu \mathrm{m}, 300$ to $550 \mu \mathrm{m}, 0.55$ to $1.0 \mathrm{~mm}, 1$ to $2 \mathrm{~mm}, 2$ to $4 \mathrm{~mm}$, and 4 to $7 \mathrm{~mm}$ ). Next, to emphasize condition-dependent details in the spatial structure of the correlations, we "corrected" the spatial pattern of pairwise correlation with respect to the stereotyped decay of correlation with increasing distance [Figs. 3(a)-3(c)]. To do so, we estimated how the average correlation varies as function of distance by fitting a polynomial to our data points from the spontaneous activity [Fig. 3(c)] and divided, for each condition, the correlation values found at any given distance by the values predicted for the same distance by this polynomial.

To compare spatial patterns of correlations between different conditions, we first increased the signal-to-noise ratio by binning pixels $3 \times 3$ and conditions $2 \times 2$ (except the blank condition, which already had twice as many repetitions as the other conditions). Then, correlations were computed for every pair of those "large pixels," averaged over trials from the same group of conditions, and "corrected" for the distance effect as described above. The results are vectors whose number of elements is the number of "large pixel" pairs. For example, Fig. 3(d) displays only subparts of these vectors, as it displays the average "corrected" correlations only for those pairs of pixels where one is the seed pixel (in white in the figure). The comparison between these vectors-which are nothing more than rearranged spatial patterns of correlation-is obtained by simply calculating the correlations between these vectors [Fig. 3(e)]: the higher the latter, the more similar are the former.

For the spectral analyses, the low-pass filtering step of the preprocessing was not performed. Spectrograms [Figs. 4(a)4(d)] were obtained by cross-correlation with a Morlet wavelet at different scales (MATLAB function cwtft). Fourier transform was applied to all signals from which the powers at different frequencies [Fig. 4(e)] could be directly obtained and were as above averaged over pixels and trials. For correlations at different frequencies [Fig. 4(f)], one has to consider that the correlation between two sine waves is equal to the cosine of their phase lag: we, therefore, extracted phase lag at individual frequencies for every pair of pixels (as the difference between the angle of complex Fourier values) and took the cosine. Then again, these correlation values were averaged over pixel pairs and trials.

\section{Results}

\subsection{Voltage-Sensitive Dyes imaging Data Recorded in V4 of the Awake Macaque Monkey}

Our study aimed at comparing the spatiotemporal structures of spontaneous and sensory-driven activities in extrastriate visual area V4 [Figs. 1(a) and 1(b)], a region that is involved in vision and visual attention (for a review, see Ref. 25), but whose organization is known in somewhat less detail than earlier visual areas, such as V1. We recorded population activity using VSD imaging, ${ }^{26}$ which allowed us to record from a large area of several square millimeters of cortex at a spatiotemporal resolution of, respectively, $30 \mu \mathrm{m}$ and $10 \mathrm{~ms}$ [Figs. 1(c)-1(f), see Sec. 2].

An awake macaque monkey trained for fixation was presented with a set of drifting luminance or isoluminant gratings at different contrast levels (see Sec. 2). The contrast of the isoluminant gratings (also called "color gratings" in the following) was purely (in the sense of the standard CIE1931 observer) chromatic (red-green), and the set of stimulus contrast values were chosen so as to evoke the same RMS cone contrast as corresponding luminance gratings (the maximal contrast displayed in conditions "luminance-100" and "color-100" had an RMS cone contrast of $18.2 \%$ - close to the maximum allowed by the color gamut of our CRT monitor). At the onset of each trial, a small fixation dot appeared on the screen-both in the "blank" (i.e., no stimulus) and stimulated conditionsand remained on until the end of a trial. In stimulated trials, a grating was displayed for $0.6 \mathrm{~s}$ after a delay of $100 \mathrm{~ms}$. In the blank trials, the screen remained uniformly gray (except for the presence of the fixation dot), as during the intertrial and interstimulus times. All stimuli, i.e., luminance and isoluminance gratings, as well as the blank and interstimulus screens, were centered on the same luminance and gray color.

Figures 1(d) and 1(e) show examples and average responses from these 13 different conditions. In our comparisons of cortical dynamics during stimulated trials and spontaneous activity, we separated the effects of the average evoked response from those of trial-to-trial remaining variability ("noise"): all individual trial time courses belonging to a given condition were divided by the time course averaged over all trials of the same condition, yielding "noise signals" [Fig. 1(f)], which we also refer to as "normalized signals" in the following. Some components of this noise were removed, as they are known to be dominated by camera noise (high-frequency component above $25 \mathrm{~Hz}$ ) or slow hemodynamic signals (linear drift, see Sec. 2). The 600-ms stimulation time period was divided into two parts: the first $250 \mathrm{~ms}$ corresponding to the "response onset" and the remaining $350 \mathrm{~ms}$ corresponding to its "plateau."

It is common practice to investigate the structure of the noise in sensory-evoked responses by quantifying variability between trials ("intertrial variability") in terms of Fano factor and 
(a)

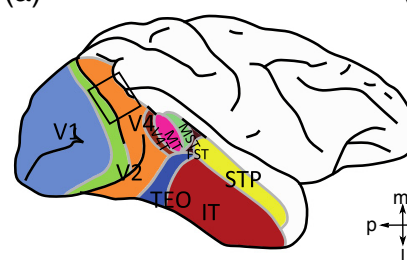

(b)

(d)

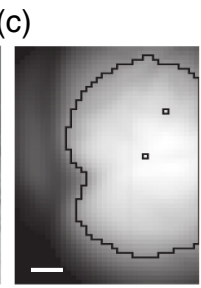

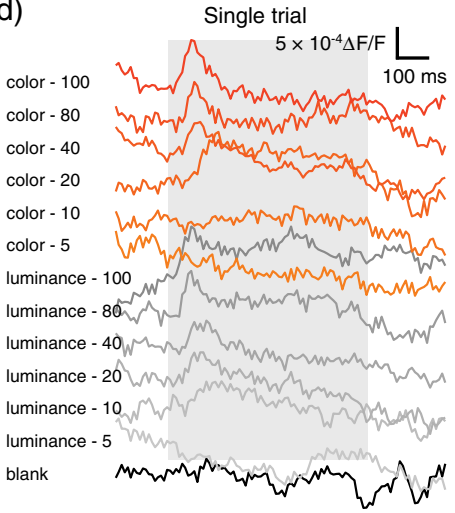

(e)
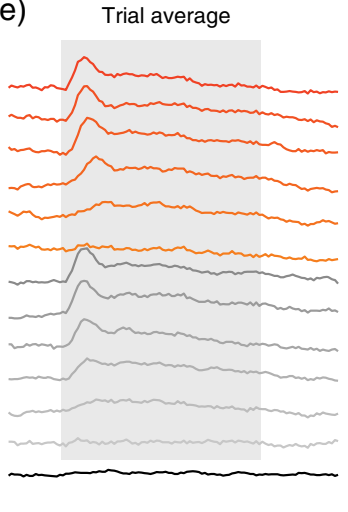

(f)
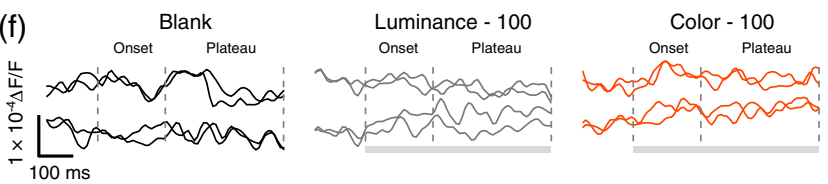

Fig. 1 VSD imaging data. (a) The recording window was centered on the dorsal part of V4 of an adult rhesus monkey. (b) Image of the surface vasculature obtained upon illumination with green $(540 \mathrm{~nm})$ light. Scale bar in (b) and (c): $1 \mathrm{~mm}$. (c) Fluorescence image of VSD. For the analysis, the camera pixels were binned $3 \times 3$ to yield $150-\mu \mathrm{m}$ sized data pixels. To exclude noisy pixels from the analysis, the latter was restricted to pixels lying within the black contour, i.e., to a region where fluorescence intensity was large. (d) Example time courses for one individual trial of each of the 13 different conditions: "color" (i.e., isoluminant red-green) gratings at six different contrasts, luminance gratings at six RMS cone contrasts equivalent to the color ones, and gray screen presentation, called "blank." Signals were obtained by averaging all pixels within the mask in c, and after removal of bleaching, heartbeat, and motion artifacts. The gray shading shows the time of the stimulation, from 100 to $700 \mathrm{~ms}$ after data acquisition start. (e) Time courses averaged over all trials belonging to each given condition. (f) Two examples of "noise" or "normalized" signals, i.e., individual trial time courses normalized by dividing them, point by point in time, by the response average over all trials belonging to the corresponding condition (additionally, signals were high-pass filtered at $25 \mathrm{~Hz}$ and detrended). Bottom and top pairs of traces correspond to two different trials; within each pair, the traces were obtained from two different pixels [squares in (c)]. The dashed line splits the stimulation period into two phases: response "onset" 100 to $350 \mathrm{~ms}$ and response "plateau" 350 to $700 \mathrm{~ms}$. Here and in all other figures, the contrast values of the visual stimuli are expressed in \% of the maximal color gamut attainable by the CRT screen.

correlations between signals recorded at different spatial locations. ${ }^{12,15,19}$ However, such quantifications are problematic when analyzing VSD imaging signals because of a number of slow drifts in the fluorescent signals (with a time constant in the order of the duration of a trial or slower) due, e.g., to bleaching or hemodynamic responses. Because of these drifts, the time course of each individual trial needs to be normalized by the average of its first frames, such that the signals express changes relative to these first frames rather than "absolute" population activity.
Despite the above shortcomings, however, VSD imaging is well suited to quantify fast activities, in frequency domains above $1 \mathrm{~Hz}$, at the population level at high spatiotemporal resolution. We focused our analysis on the study of the spatiotemporal characteristics of individual trials, and later averaged the resulting quantifications over all trials during which the same stimulus condition had been used.

\subsection{Decrease in Temporal Variability and in Spatial Correlation During a Sensory Input}

We first measured the power of the noise signal time courses, i.e., the RMS of the variability over time of the individual pixel values, and then averaged over all pixels within a mask [Fig. 1(c)] and over all trials from the same condition. We found that on the average, the power of noise signals is lower during the plateau period of stimulated conditions than during spontaneous activity [Fig. 2(a)]. This result also holds true without normalization by the average responses (not shown). During the response onset period, on the contrary, temporal variability was found to be higher than during spontaneous activity. This was probably due to an ineffective removal of the evoked responses by the average response normalization procedure because of large intertrial differences in onset delay and amplitude [not shown, but see later comments on Fig. 4(b)].

We then measured the correlation between the noise signal time courses recorded from pairs of pixels [e.g., the signals shown in Fig. 1(f), which originate from the pixels as shown in Fig. 1(c)] and averaged over all pairs and trials [Fig. 2(b)]. As in the case of the time courses' power, their pairwise correlations during the plateau period of stimulated conditions were on the average lower than during spontaneous activity. However, this decrease in correlation was considerably more pronounced than that of temporal variability (on average $-37.4 \%$ versus $-6.9 \%$, in relative units). To better visualize this result and the relation between these two variables, we displayed the spatial activity patterns appearing in the noise signal along one spatial dimension as a function of time [Fig. 2(c)], both for stimulated and blank conditions. Whereas global activity patterns (in space) did occur both in the case of spontaneous and sensory evoked activity, they occurred more often in the former case as can be seen qualitatively in Fig. 2(c). The same result is confirmed quantitatively by the distributions of correlation values in Figs. 2(d)-2(e) (note that the very same trials had high variability and high correlation values).

With respect to more traditional definitions of intertrial variability and noise signal correlations, our quantification of variability and correlation in the time domain have the advantage of not being affected by the slow drifts present in VSD imaging signals. In addition, they allow detailed characterizations in the spectral domain (see Sec. 3.4). In Appendix A, we develop the mathematical details of both approaches and how they relate to each other. Indeed, intertrial variability could not be properly evaluated for our VSD imaging data due to such slow drifts. Fig. 2(f) quantifies the variability over trials of the noise signal as RMS, averaged over the plateau period and over all pixels: the average RMS of the intertrial variability value, some $5 \times 10^{-4}$ [Fig. 2(f)], is more than twice as large as the average RMS of the temporal variability [Fig. 2(a)]. This large noise hampers the comparison of the intertrial variability of different conditions. 
(a)
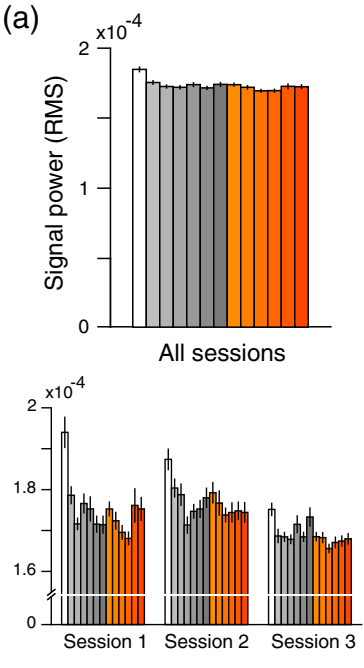

(d)

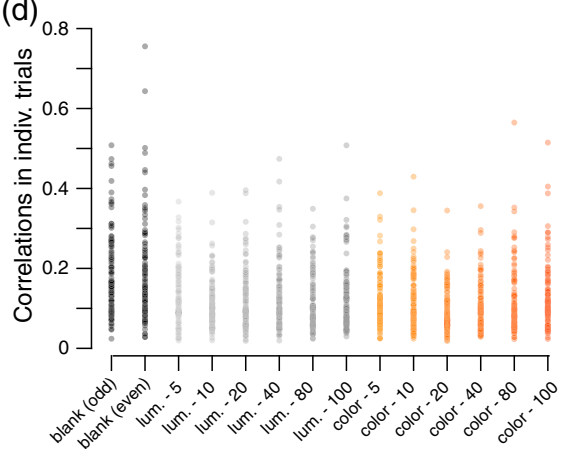

(b)
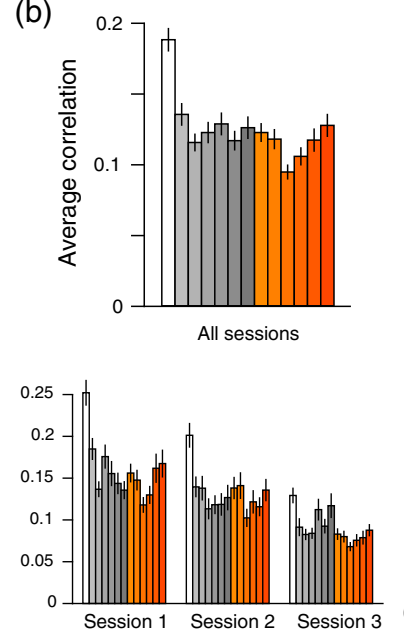

(e)

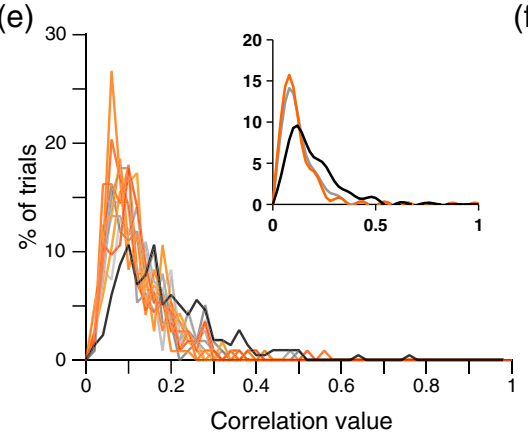

(c)
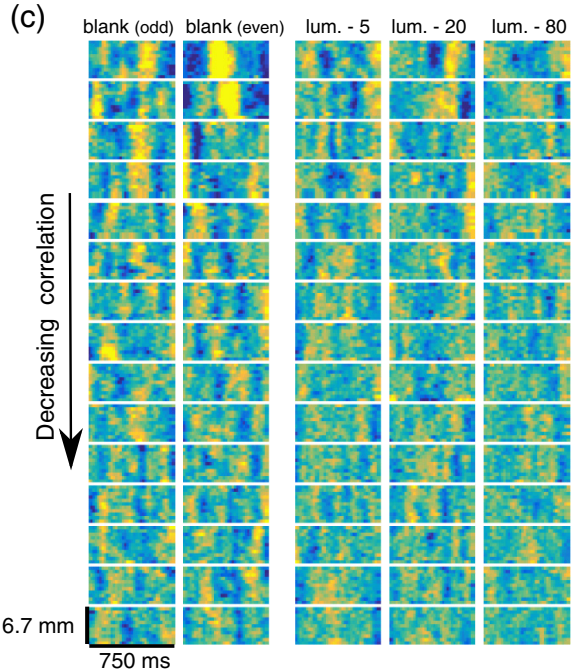

color -5 color -20 color -80

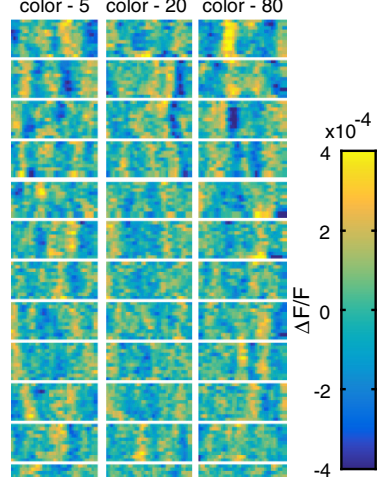

Fig. 2 Variability and correlation during the response plateau period. (a) Variability over time of the noise signals [the same processing as in Fig. 1(f)], calculated as RMS power during the plateau period and averaged over all pixels within each session's specific mask [Fig. 1(c)], and over all trials pooled from the three sessions. Error bars: standard error calculated over the pooled trials. In every session, the power of the normalized signal was larger during spontaneous activity than during stimulus-driven activity. (b) Correlations between the noise signals recorded from pairs of pixels during an individual trial's plateau period were averaged over all pixel pairs and all pooled trials. Correlations are significantly lower when calculated during sensory driven than during spontaneous activity. (c) Spatiotemporal activity patterns recorded during the plateau period of individual trials (for the spatial coordinate, a vertical line is used crossing V4 and passing through its center), sorted by increasing contrast (left to right) and by decreasing correlation (top to bottom). Although low spatiotemporal frequency patterns appear both in the blank and in the stimulated conditions, they are more frequent in the former compared with the latter (as twice as much blank trials than stimulated ones were collected, they were separated into odd and even ones and displayed, as in (d), as two independent conditions to provide a fair comparison with the stimulated conditions). (d) Plot showing the average pairwise correlation for each trial and each condition. Again, high correlation trials were more frequent during the blank than during the stimulated conditions. (e) Histogram of correlations (orange, color; gray, luminance; black, blank). Inset: smoothed histogram of trials grouped according to luminance, color, and blank, showing a clear shift of the blank distribution toward higher correlations. (f) Trial-to-trial variability. RMS over trials were calculated [the same processing as in Fig. 1(d), i.e., no normalization] and averaged over all pixels within the mask and over the whole duration of the plateau period. No clear result could be observed, as a probable consequence of slow drifts in the VSD signals, with a time constant in the order of the intertrial interval. Here and in all other bar displays of this work, darker gray and increasingly saturated red signify increasing stimulus contrast. The white bar stands for the unstimulated condition.

One may wonder if the decrease of a global signal alone can account for the strong $-37.4 \%$ decrease in correlation while representing only a mild $-6.9 \%$ decrease in total power, or if on the contrary, a concomitant increase in uncorrelated (or only locally correlated) signals is needed to explain such a weak power drop. We treated this question analytically (somewhat simplifying the real case assuming two regions of interest only: see Appendix B for details) and found that a decrease in global signal alone is indeed compatible with our data. As a side note, we even found that, at average correlation values as low as 0.2 , the ratio between correlated and uncorrelated activities is such that decreases in global activity can lead to 8 times larger decreases in correlations. We stress, however, that the measured signals are contaminated by an important photonic noise, which is by essence uncorrelated. Therefore, the "true" membrane potential signals are likely to have higher average correlations than 0.2 , 
and thus to experience smaller correlation drops than the ones we observe in our signals, given the same reduction in global activity.

\subsection{Spatial Structure of Correlations}

Next, we further characterized the spatial structure of the correlations between the noise signals recorded from different locations and their changes across conditions. The most prominent spatial feature is a decrease of correlation with distance [Fig. 3(a)], which, modulo a multiplicative factor most likely due to the different kind of recorded signals (multiunit versus VSD imaging), is - in relative terms-compatible with the decline reported by Ref. 21 .

To investigate how correlation changes across conditions at different spatial scales, we thus grouped pixel pairs according to their distance. Figure 3(b) and Table 1 show that these changes were rather similar at all spatial scales, with a slight tendency of the relative difference in correlation between evoked and ongoing activity to increase with distance between the points for which the correlation was calculated.

The decrease of pairwise correlation with distance creates a strong spatial pattern. We thus wondered whether it might not be masking weaker, condition-dependent, spatial structures. To access such finer structures, we estimated how the average correlation varies as a function of distance by fitting a polynomial to our data points from the spontaneous activity [Fig. 3(c)] and corrected the correlation values for this distance effect in all conditions (see Sec. 2). To increase the signal-to-noise ratio, we moreover binned the pixels $3 \times 3$ and grouped the condition contrasts $2 \times 2$. Examples of the resulting "average correlation corrected" maps are shown in Fig. 3(d), each displayed at its own color scale based on its mean and standard deviation (std.) to get rid of the overall correlation decrease in the stimulated conditions with respect to the spontaneous case.

Figures 3(b) and 3(d) show that, indeed, correlation did have a specific spatial structure. Note that this structure was similar throughout conditions: it can, therefore, not be attributed to random noise. Moreover, the example shown for one seed pixel in Fig. 3(d) suggests a higher similarity of the spatial structure of correlations among stimulated conditions than between any stimulated condition and spontaneous activity. We, therefore, quantified this similarity between spatial correlation patterns using a distance (being itself based on correlation, see Sec. 2) and averaged over all seed pixels. The results are displayed in Fig. 3(e) and confirm and generalize what is shown in Fig. 3(d): after average over all seed pixels, the "fine spatial structure" of correlations between sensory-evoked activities in different locations is more similar among different stimulated conditions than it is between a stimulated and a nonstimulated one.

\subsection{Spectral Structure}

To further characterize the differences in temporal variability and correlations between spontaneous and evoked activity, we investigated the signals' various frequency components. First, we performed time-frequency analysis of the raw [i.e., nonnormalized as in Fig. 1(d)] signals using a wavelet transform [Figs. 4(a)-4(b)]. With respect to spontaneous activity, the evoked activity displayed two main characteristics. First, a clear increase in power is in the range of 5 to $10 \mathrm{~Hz}$ during the response onset phase- - the larger the increase, the stronger (a)

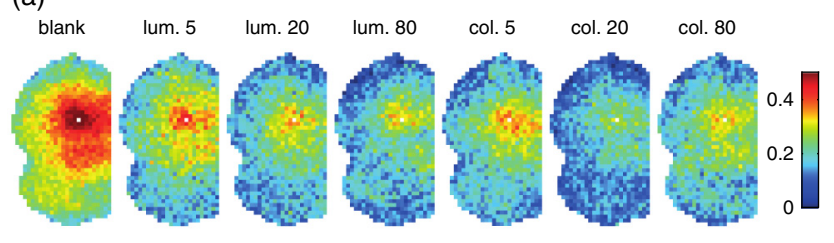

(b)
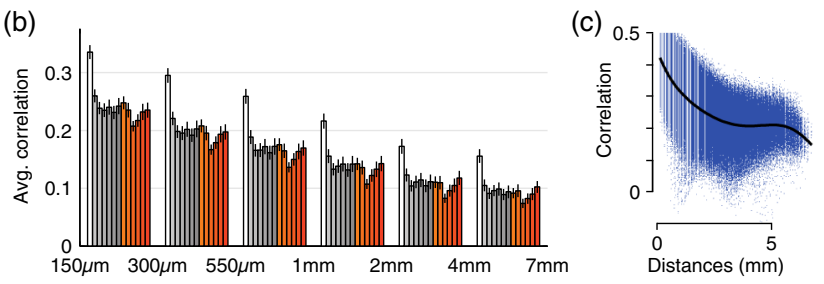

(d)

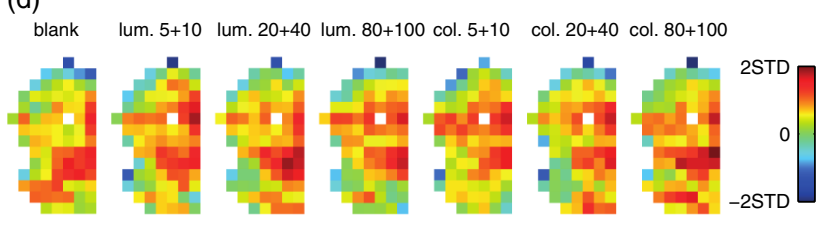

(e)
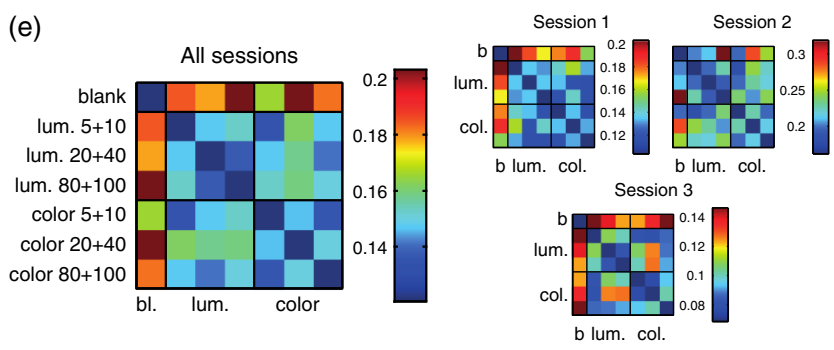

Fig. 3 Spatial analysis. (a) Example maps of the correlations between a seed pixel and all pixels within the mask [see Fig. 1(c)], during the plateau period of the normalized signals, for a subset of the used contrast levels (data from session 1). As expected, correlation decreases with distance and is altogether lower in the case of sensory-driven activity than during blank. (b) Correlations averaged over pixel pairs grouped according to their distance range (average result over all sessions). (c) Polynomial fit of the correlation during the blank stimulus as a function of distance between pixels. Each point represents one pixel pair from the same session as in a (each session was fitted independently, shown data stem from session 1). (d) Fine structure in correlation patterns (the same session as shown in a); to increase signal-to-noise ratio pixels were grouped $3 \times$ 3 and condition contrasts were grouped $2 \times 2$. Moreover, the distance effect on correlation was removed by normalizing by its estimation (see c); finally, to get rid of the global correlation decrease in stimulated conditions, we display the correlation maps after normalization by their mean and std. (i.e., conversion into $z$-scores). (e) Distances between correlation patterns of the different conditions. As shown in a more graphical way already in (d), the correlation maps obtained from the stimulated conditions are more similar one to another than they are to that obtained from spontaneous activity.

Table 1 Relative difference in correlation between the spontaneous activity and the evoked activity at various spatial scales. Positive values indicate a correlation drop with respect to spontaneous activity.

\begin{tabular}{|c|c|c|c|c|c|c|}
\hline & $\begin{array}{c}150 \text { to } \\
300 \mu \mathrm{m}\end{array}$ & $\begin{array}{l}300 \text { to } \\
550 \mu \mathrm{m}\end{array}$ & $\begin{array}{c}0.55 \text { to } \\
1 \mathrm{~mm}\end{array}$ & $\begin{array}{c}1 \text { to } \\
2 \mathrm{~mm}\end{array}$ & $\begin{array}{c}2 \text { to } \\
4 \mathrm{~mm}\end{array}$ & $\begin{array}{c}4 \text { to } \\
7 \mathrm{~mm}\end{array}$ \\
\hline Correlation (\%) & 30 & 34 & 36 & 37 & 38 & 41 \\
\hline
\end{tabular}


(a)

(b) Raw data
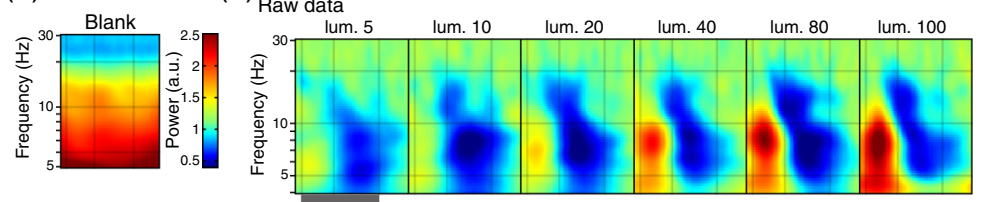

(c)

(d) Normalized data
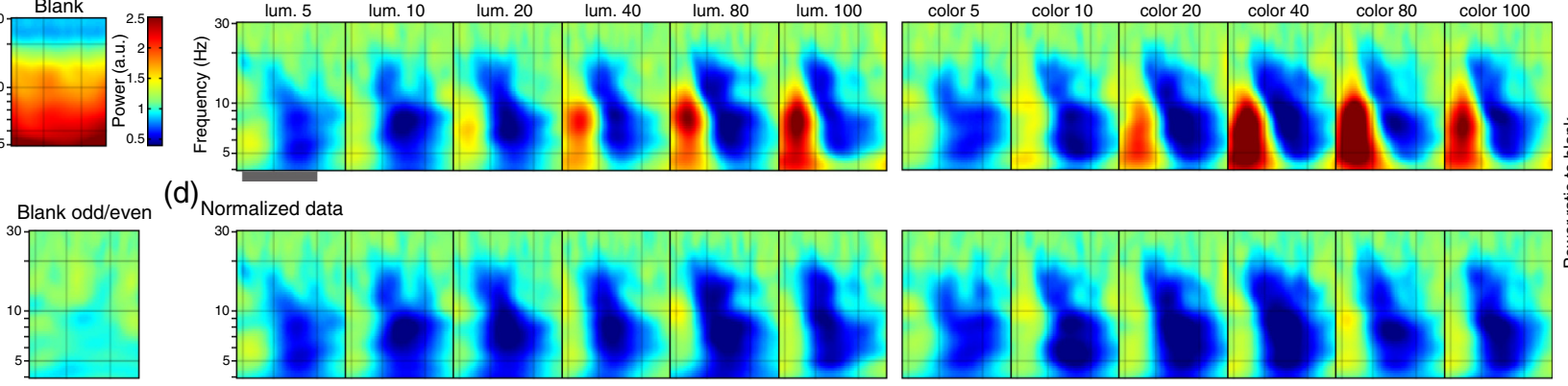

(e)

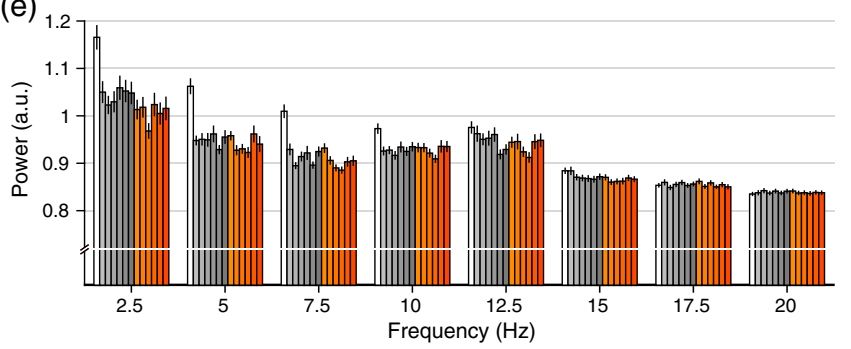

(f)

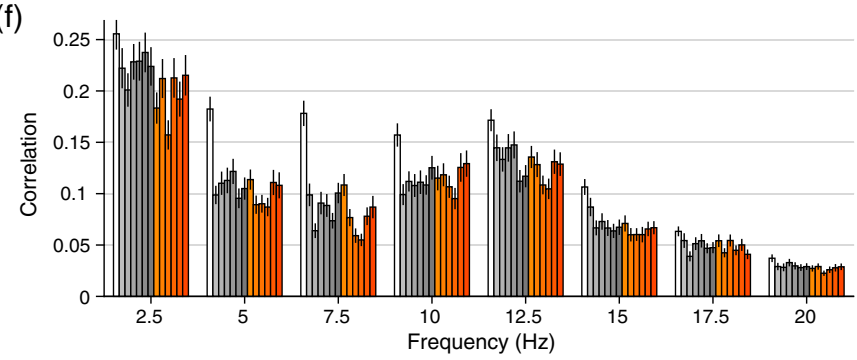

Fig. 4 Time-frequency analysis. (a-b) Raw signals. Spectrograms were computed for every condition and averaged over pixels, trials, and sessions. The $x$ - and $y$-axes represent time in $\mathrm{s}$ [the 600-ms stimulation period in (b) is marked by the gray bar and the grid] and frequency in $\mathrm{Hz}$, respectively. The image in a shows the time-frequency decomposition of spontaneous activity (blank trials). Panel (b) shows the time-frequency decomposition of evoked activity (stimulated trials), relative to the spontaneous one (i.e., after division by a). To give an indication of the level of noise, the image in (c) compares odd and even blank trials. Two main phenomena can be observed in (b): an increase in the range of 5 to $10 \mathrm{~Hz}$ component's power during the response onset and a decrease in the range of 5 to $15 \mathrm{~Hz}$ component's power during the plateau period at all contrast. (d) the same as (b), but on what we call the "noise signals," i.e., on the signals normalized by the trial-averaged signal. Note that most of the positive response [red in (b)] is lost due to the removal of the average stimulus-evoked response by the normalization. (e-f) Bar display of power and correlation calculated on the normalized signals during the plateau period, selectively for different frequency bands [unlike in (b) and (d), without division by the spontaneous activity]. These two quantities decrease during sensory-driven activity, in particular in the range of 5to $12.5 \mathrm{~Hz}$.

the stimulus contrast. Second, we observed a power decrease in the range of 5 to $15 \mathrm{~Hz}$ during the plateau period, the amplitude of which was independent of stimulus contrast.

When the same wavelet transform was applied to the "noise" signals (i.e., after normalization by the average response for a given condition), the increase at 5 to $10 \mathrm{~Hz}$ during the response onset was largely reduced as expected [Figs. 4(c)-4(d)]. The small rests of this increase in signal power are probably due to some intertrial variability in the delay and amplitude of the individual response onsets, which prevent their complete removal by average response normalization. On the other hand, the decrease of activity in the range of 5 to $15 \mathrm{~Hz}$ not only remained but was even more pronounced after normalization and far beyond the noise level [quantified in Fig. 4(c)].
For a more rigorous quantification of stimulus-induced changes in the noise signals, we calculated their Fourier transform restricted to the plateau period. In this way, one gains access to power [Fig. 4(e)] and correlation [Figs. 4(f) and $4(\mathrm{~g})]$ in different frequency bands (see Sec. 2). Table 2 summarizes the observed differences between spontaneous activity and the average over all stimulated conditions. Temporal variability (expressed here as Fourier power) was lower in the case of evoked activity compared with spontaneous activity, in particular in the 2.5 to $10 \mathrm{~Hz}$ band, with a maximal relative difference of $12 \%$ at $2.5 \mathrm{~Hz}$. The same was observed for correlation (for details on calculations, see Sec. 2), with an even larger peak drop of $55 \%$ at $7.5 \mathrm{~Hz}$, even at the lowest contrasts. Together, Figs. 4(e) and 4(f) quantify what can be already

Table 2 Relative changes in power and correlation between the spontaneous and evoked activities at different frequencies (the same sign conventions as in Table 1).

\begin{tabular}{lcccccccc} 
& $2.5 \mathrm{~Hz}$ & $5 \mathrm{~Hz}$ & $7.5 \mathrm{~Hz}$ & $10 \mathrm{~Hz}$ & $12.5 \mathrm{~Hz}$ & $15 \mathrm{~Hz}$ & $17.5 \mathrm{~Hz}$ & $20 \mathrm{~Hz}$ \\
\hline Power (\%) & 12 & 12 & 10 & 5 & 3 & 2 & 0 & 0 \\
Correlation (\%) & 18 & 44 & 55 & 30 & 26 & 37 & 24 \\
\hline
\end{tabular}


(a)

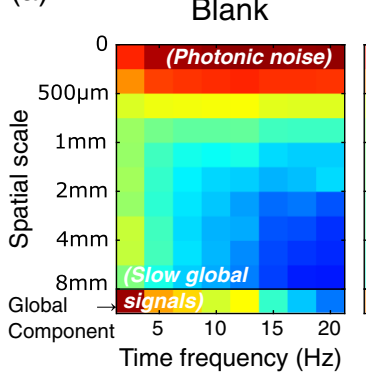

Stimulated

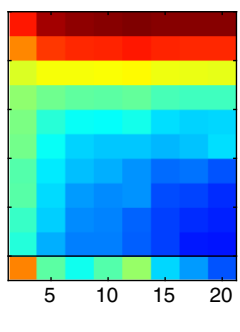

(b)

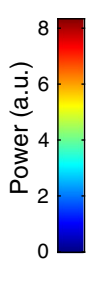

Stimulated / blank

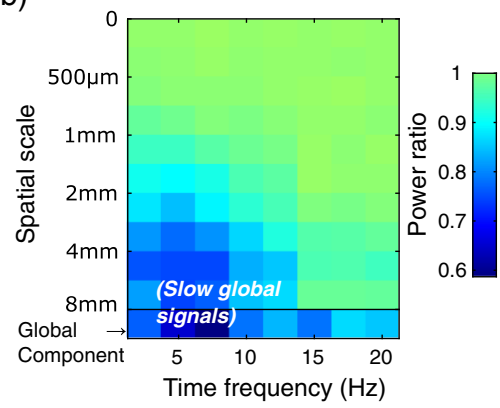

(c)

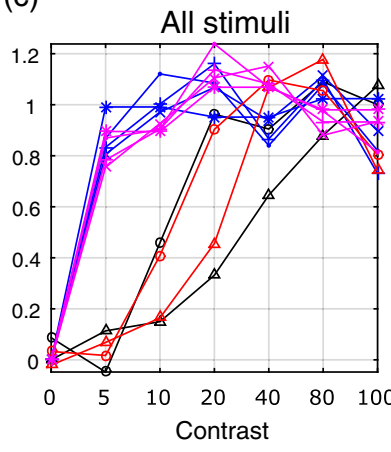

(e)

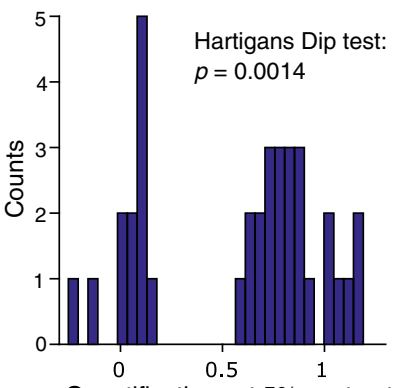

(f)

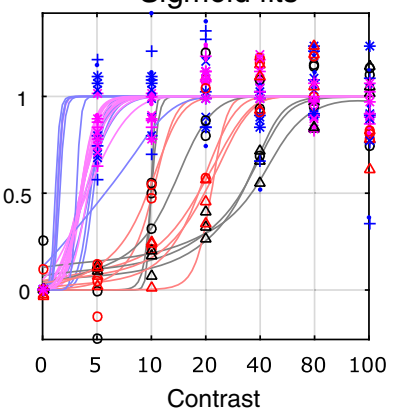

(d) Individual stimuli, all sessions

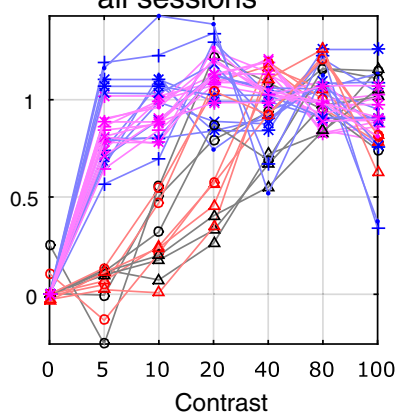

(g)

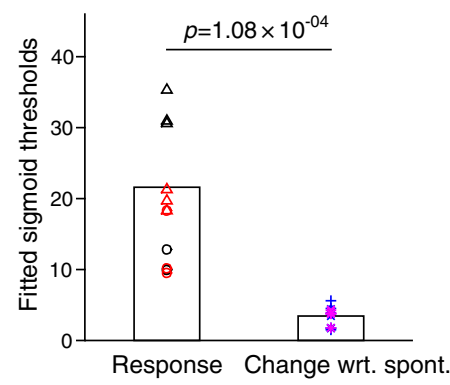

Fig. 5 Synthesis. (a) Spatiotemporal power spectrum of the noise signal during blank and stimulated conditions. Each spatiotemporal bin sums the power values contained in the specified temporal and spectral intervals. Note the large, high temporal frequency and spatially uncorrelated shot-noise component, as well as the global components in the low spatial frequency range. (b) Power ratio, showing the reduction of low spatial and mid-low (alpha range) temporal frequency components in stimulated versus blank conditions. (c) Different contrast-response dynamics, average over trials and sessions: quantities that change with stimulus contrast were normalized between 0 and 1 (based on the baseline and plateau of the fitting sigmoids) and displayed together, (o) the amplitude of the average responses [Fig. 1(e)], averaged during the response peak, i.e., between 230 and $300 \mathrm{~ms} .(\Delta)$ the initial, positive, responses in the range of 5 to $10 \mathrm{~Hz}$ [Fig. 4(b) and 4(d)]. (+ and $x$ ) the decrease in temporal variability [Fig. 2(a)], in correlation [Fig. 2(b)] and in power in the range of 5 to $15 \mathrm{~Hz}$ [Figs. 4(b) and 4(d)], during the plateau period. $\left(^{*}\right)$ the differences in the spatial patterns of correlation found during spontaneous activity and during stimulated conditions [Fig. 4(f)]. Two main different contrast response functions can be observed: on the one hand, response amplitudes start to increase at contrast $10 \%$ and saturate between contrast $20 \%$ and $80 \%$; on the other hand, the changes in variability and correlation are already saturated or close to saturation at contrast $5 \%$ and the same is true for the power undershoot in the range of 5 to $15 \mathrm{~Hz}$ band. (d) as (c), but for single sessions (average over trials). (e) $5 \%$ contrast response histogram for all trials and classes of cortical variables, showing a clear bimodal distribution: each count in the left or right clusters corresponds, respectively, to one of the red-black or the magenta-blue curves in (d)-(f) Sigmoid fits to the data in (d). (g) The difference in the $50 \%$ sigmoid contrasts observed between trials belonging to the first class of variables ["raw response amplitudes," red and black in (c, d, f)] and the second class ["difference with respect to blank," magenta and blue in (c, d, f)] is statistically highly significant. 
seen qualitatively in Fig. 2(c): at the population level, during the stimulated conditions, the blobs of activity patterns tend to become smaller in both the spatial dimension (reduced correlation) and in the temporal one (less low-frequency components) compared with the blank conditions. A summarizing comparison of the spatiotemporal dynamics during visual stimulation and the blank is shown in a synthesis view in Figs. 5(a)-5(b).

\subsection{Different Classes of Variables have Different Contrast Response Gains}

Our results show that the sensory-evoked decrease of variability (the power) of "noise" signals and their spatial correlation reached saturation at very low contrast values, if not at the lowest one [Figs. 2(a)-2(b), 3(b)-3(c), 3(e), 4(d)-4(f)]. The contrast dependency of the average raw responses was strikingly different [e.g., see Fig. 1(e)]: there, a rather gradual amplitude increase was observed, starting from weak, if any, response to the lowest stimulus contrast, increasing gradually and saturating only at the highest contrast values, if at all [Fig. 5(c)].

To further investigate this difference in contrast gains, we analyzed the distribution of the various variables' contrast dynamics for the individual sessions [Fig. 5(d)]. Like the grand average responses in Fig. 5(c), the single session ones also split into two populations, corresponding to the cortical activity variables mentioned above: an abrupt decrease in "noise signal" (i.e., temporal variability) and its alpha-range power, as well as an abrupt drop in its pairwise correlations and their spatial difference with respect to the blank (magenta and blue in Fig. 5), as opposed to the amplitude of the raw responses, which changed only gradually with stimulus contrast (red and black in Fig. 5). At the critical contrast level of 5\%, where the differences between the various variables were largest, the distribution of their contrast dynamics was clearly bimodal even upon considering simply the raw data points [Fig. 5(e)], yielding a $p$ value of $1.4 \times 10^{-3}$ when tested against a unimodal distribution (Hartigan's dip test). To test whether there were also two different contrast dynamics over the full contrast range (and not only at the 5\% contrast value that displayed clear bimodality), we first fitted the data points in Fig. 5(d) with sigmoids [Fig. 5(f)]. Then, we compared their $50 \%$ contrast values by separating their populations into the two classes [left and right columns in Fig. 5(g)] obtained from the histogram in Fig. 5(e). Again, the difference between the distribution split into two populations in a highly significative way $\left(p\right.$ value $=1.08 \times 10^{-4}$ ), strongly supporting the notion of two different classes of cortical activity variables.

We summarized these facts in Figs. 5(c)-5(g), which indeed show two different shapes of contrast response functions, both at the grand average level and at the individual session level. A first one, that applies to different quantifications of the (raw) response onset, begins to increase at contrast value 10 and saturates between contrast values 20 and 80 (somewhat depending on the type of stimulus and the particular response quantification). The second one, that applies to the changes in temporal variability of the noise signals, their average spatial correlation, and correlation structure, as well as to the delayed alpha band ( 5 to $15 \mathrm{~Hz}$ ) undershoot in both the raw and the noise signals, essentially saturates already at contrast value 5 .

\section{Discussion}

\subsection{Spatiotemporal Characterization of Spontaneous and Sensory-Driven Cortical Dynamics Using Voltage-Sensitive Dyes Imaging}

As opposed to previous studies that mostly focused on intertrial variability and that reported its reduction as a result of sensory input, ${ }^{9-14}$ here, we characterized the changes in spatiotemporal dynamics of cortical activity occurring during sensory inflow, in full detail and at high resolution in cortical area V4 of an awake macaque monkey.

Our first - and perhaps most important — result is that a particular type of activity is reduced during sensory inflow, namely slow-wave activity with prominent power at spatial scales larger than $1 \mathrm{~mm}$ and temporal frequency in the alpha range and below [Figs. 5(a)-5(b)]. In addition, we propose that this phenomenon is at the origin of the decrease in variability reported both by ourselves and by others, in the temporal sense (our "noise signal") as well as in the intertrial sense ${ }^{9-14}$ (under some reasonable assumptions and for large trial numbers the two actually coincide, see Appendix A). Importantly, such a reduction of spatially global activity can also explain the sensory-evoked reductions in pairwise correlations detected both in the present study and by others ${ }^{21}$ (at least at first-order approximation, see the next section).

Such results emphasize the relevance of wide-field VSD imaging for studying the spatiotemporal structure of cortical activity. Indeed, although lacking single-cell resolution, VSD imaging is ideally suited for imaging the activity of comparatively large neuronal populations in vivo, providing an excellent combination of temporal and spatial resolution (in our hands: $110 \mathrm{~Hz}$ and, laterally, some $30 \mu \mathrm{m}$, which is clearly finer than the typical dimensions of cortical columns) and field of view $\left(750 \mathrm{~ms},>10 \mathrm{~mm}^{2}\right)$. Moreover, VSD imaging is complementary to (multi-)electrode recordings, first, because it does not suffer from sampling issues (at least not the same ones as multielectrode recordings: VSD imaging indeed lacks depth resolution/penetration); second, it also provides different information on the local network activity. Indeed, the VSD imaging signal reflects the average membrane potential changes of the neuronal processes below a given pixel and is, therefore, biased toward information processing by the local network rather than toward the network's output-as is the case when recording spikes (for a biophysical modeling of the VSD imaging signal, see Ref. 27). On the other hand (and differently from electrophysiological recordings), VSD imaging signals are more suitable to record relative changes in activity rather than absolute ones. Indeed, even if the relation between membrane potential and fluorescence is assumed to be linear, ${ }^{28,29}$ the parameters (offset and slope) of this linear relationship are not only unknown but may also change from one pixel to another as well as during the course of the experiment (although slowly). This makes it problematic to extract absolute activity values from the VSD imaging signals such as absolute membrane potentials or numbers of spikes. VSD imaging is thus less suitable to investigate signal changes slower than $0.1 \mathrm{~Hz}$, as is needed in the case of intertrial variability, which could indeed not be investigated with the methods used here [Fig. 1(f)]. 


\subsection{Mere Reduction of Specific Activity or Network Reconfiguration?}

Although in our data, the most obvious change in network activity induced by sensory input appears to be the reported decrease in slow global activity, one may wonder whether other, more complex, changes also occur. In particular, is the drop in spatial correlation entirely due to decreased global activity or does uncorrelated activity increase, too?

Our theoretical investigation (Appendix B) showed that, at an average correlation value of 0.2 , the observed decrease in global (and therefore correlated) activity ( $-6.9 \%$ in average) is-in principle - enough to account for the large decrease in pairwise correlations $(-37.4 \%)$.

However, the above theoretical result by no means rules out specific reconfiguration to occur in the network. On the contrary, when we visualized spatial maps of correlation [after subtraction of the stereotyped pattern due only to distance, Fig. 3(c)], we observed clear sensory-evoked modifications of the spatial patterns of correlation, with respect to those observed during ongoing activity [Figs. 3(d)-3(e)]. This suggests that more complex changes in spatiotemporal dynamics occur upon sensory input than a simple global activity decrease. This also reconciles our results with previous findings that spontaneous activity is spatially structured. ${ }^{7}$ Spontaneous activity could thus be thought of, at the first order, as global slowwave activity, and at higher orders as endowed with finer structures riding on top of it, probably involving specific neuronal connections.

Detailed insight on noise dynamics and beyond has recently been provided within a Bayesian framework by the modeling work of Ref. 16 for neuronal processing in primary visual cortex, lacking, however, experimental testing on certain aspects. Our data could provide those. Notably, our data appear to confirm the predicted within-trial variability reduction a result of sensory input, thus lending support to their model predictions. Another aspect where our data would seem to join ${ }^{16}$ are the differences in spatial correlation patterns we observed between blank and stimulated conditions, which would go along with the change in interneuronal covariance predicted by the model. With this respect, it would be interesting to investigate those predictions in further detail for larger neuronal populations and more contrast values than the published ones, ${ }^{16}$ for the purpose of a more complete compatibility test with our data.

\subsection{Two Different Contrast Dynamics}

Our second main finding is that the activity changes reported above are already near-maximal at the lowest contrasts used in our experiments, displaying a dependence on contrast that is remarkably different from those of the responses themselves, which are barely visible at the lowest contrast [Figs. 5(c)$5(\mathrm{~g})]$. In other words, cortical activity variables appear to separate into two groups, characterized by different contrast dynamics: the raw responses, characterized by a fast onset and a gradual increase with stimulus contrast, as opposed to a second group of variables, characterized by a nonlinear contrast dynamics that becomes more pronounced during the plateau period of the response (or at least is more separable from the first one during this later response phase). It is tempting to speculate that this delay might be the signature of more complex changes in the spatiotemporal dynamics of local neural activity, beyond the mere feed-forward flow of information. However, such a bimodal distribution of variables characterizing cortical dynamics does not necessarily imply the existence of two distinct pathways or mechanisms in the processing of sensory information. Indeed, such behavior can be predicted by existing models of neural networks, with abrupt changes as a result of even a minimal input, e.g., due to mechanisms such as bifurcation crossing, ${ }^{30}$ by switching from multimodal stability ("ghost" attractors) a unimodal stability ${ }^{31}$ or by overall activity decrease. ${ }^{32}$ The contrast dependence of cortical dynamics seen in our data could thus serve as a benchmark for these existing models, in particular for the recent Bayesian model by Ref. 16 that includes a systematical analysis of both first- and secondorder cortical activity statistics, of the signal itself as well as of the "noise" (i.e., variability). Unfortunately, in that study, only three contrast values were treated, making it difficult to discern whether the contrast dependence of the activity variables reported in that study (variability of population responses and stimulus-specific distance between means, covariance between cells, etc.) is abrupt or rather near-linear.

\subsection{Implications for the Role of Spontaneous Activity}

Our experiments were not explicitly designed with the goal of determining the functional role of spontaneous activity or of its relation with sensory-evoked activity. Neither do our results allow to answer the question of whether the source of response variability (i.e., ongoing activity) has a function for the treatment of sensory information or whether it should rather be considered as a nuisance for sensory processing (instead of an advantage), calling for the need to be temporarily silenced not to hamper the latter. Nevertheless, as outlined below, our findings have implications for both scenarios.

Shortly, the notion that the spontaneous activity may actively participate to sensory processing was triggered among else by the pioneering works of Grinvald and colleagues that emphasized its influence on evoked responses ${ }^{2}$ and its map-like spatial structure. $^{7}$ A Bayesian formulation of perception was proposed shortly afterward, ${ }^{6,33-35}$ which, coupled with the postulate that spontaneous activity encodes some $a$ priori knowledge within the functional structure of the underlying network, offers an interesting unifying view accounting for the bidirectional interactions between spontaneous and sensory-evoked activities mentioned above (see also the review by Ferezou and Deneux in this special issue of Neurophotonics). According to this formulation, evoked activity represents an a posteriori knowledge of the visual scene, i.e., a combination of the a priori knowledge encoded in the spontaneous activity with the new information provided by the sensory input. As a consequence, evoked responses are determined not only by the sensory inflow itself but also by the ongoing spontaneous activity that encodes a priori knowledge, and the decrease of the responses' variability compared with that observed in the spontaneous regime is interpreted as a reduction of perceptual uncertainty.

In this respect, the presence of two different contrast dynamics in our data raises the following question: does the fact that some of the changes in the measured cortical dynamics are nearsaturated already at the lowest contrasts [Figs. 5(c)-5(g)] suggest that the perceptual uncertainty is already resolved, despite sensory inflow being only minimal? Put differently, does the transition in network activity as detected in VSD imaging at low contrast values correspond to a transition in perception? This hypothesis could be tested by behaviorally determining 
the monkey's contrast threshold for perceptive processing of the stimulus (preferably simultaneously with imaging), and then testing it for similarity with the contrast threshold we found with respect to a change in cortical dynamics. In case of a positive outcome, it will be necessary to model in a biologically plausible way how and what kind of a priori information on its environment the brain encodes in the slow and global activity patterns we found to be characteristic - at least at the first order-of ongoing activity, and which decrease as a result of sensory input.

An alternative view on the function of spontaneous activity is that it performs some background, low-level "maintenance" task (see Ref. 36 for a short review). Should this be the case, our results would indicate the existence of a switching mechanism that shuts down (or reduces at least) this background activity during the processing of incoming sensory information. This hypothesis was already raised in Ref. 19, which observed a decrease of up and down fluctuations in anesthetized rat barrel cortex, lasting for several seconds after a brief somatosensory stimulation. Importantly, such a switching mechanism would not need to be explicitly formulated in a model of cortical activity. Rather, it could be embedded within an intrinsic mechanism that decreases overall variability-thus also our "noise signal"- upon the entrance of a new sensory input. With this respect, theoretical work $^{32}$ has shown that-under certain conditions-sensory input can reduce variability in recurrent networks. ${ }^{32}$ Once more, however, experimental data ${ }^{11}$ show that such a reduction of ongoing activity is compatible with both interpretations of ongoing activity, underscoring the need for additional experimental work to resolve this issue.

\section{Appendix A: Relation Between "Temporal Variability" and Classical Intertrial Variability, Noise Correlations}

Let us note $a_{x, t, k}$ the optical signal recorded in pixel $x$, at time $t$, during the trial $k$. We first convert these raw data into what we call "noise signal," i.e., the fluctuations of the signal with respect to the mean over trials, normalized by the latter

$b_{x, t, k}=\left(a_{x, t, k}-\left\langle a_{x, t, k}\right\rangle_{k}\right) /\left\langle a_{x, t, k}\right\rangle_{k}$.

Our "temporal variability" is then computed as the noise signal's RMS, averaged over trials and pixels, i.e., $\left\langle\sqrt{\left\langle\left(b_{x, t, k}-\left\langle b_{x, t, k}\right\rangle_{t}\right)^{2}\right\rangle_{t}}\right\rangle_{k, x}$ and is a scalar.

Classical, customary "intertrial variability" is computed as $\left\langle\sqrt{\left\langle b_{x, t, k}^{2}\right\rangle_{k}}\right\rangle_{x}$ and is a function of $t$ (although it can obviously be averaged over time).

In the general case, one cannot predict one value from the other, but if

1. the activity is assumed to be stationary over a particular time segment (e.g., a segment of spontaneous activity or the plateau period of the response), i.e., for every time $t$ in this segment and for every trial $k, a_{x, t, k}$ follows the same law with mean $\bar{a}$ and variance $\sigma^{2}$, and

2. this time window is significantly longer than the typical correlation distance between different time instants, such that $\left\langle b_{x, t, k}\right\rangle_{t}$ is close to zero, and the expectation of $\left\langle\left(b_{x, t, k}-\left\langle b_{x, t, k}\right\rangle_{t}\right)^{2}\right\rangle_{t}$ is close to $(\sigma / \bar{a})^{2}$, then both computations converge to the same result $\sigma / \bar{a}$ when the number of trials goes to infinity.

Importantly, only our computation allows us to investigate the variability's power in specific frequency bands (and to deal with the problem of the slow baseline fluctuations that typically affect VSD imaging signals).

For the correlation, our correlations between two pixels $x \neq x^{\prime}$ are computed using time as the internal variable and then averaged over all pixel pairs and trials:

$\left\langle\frac{\left\langle\left(b_{x, t, k}-\left\langle b_{x, t, k}\right\rangle_{t}\right)\left(b_{x^{\prime}, t, k}-\left\langle b_{x^{\prime}, t, k}\right\rangle_{t}\right)\right\rangle_{t}}{\sqrt{\left\langle\left(b_{x, t, k}-\left\langle b_{x, t, k}\right\rangle_{t}\right)^{2}\right\rangle_{t}} \sqrt{\left\langle\left(b_{x^{\prime}, t, k}-\left\langle b_{x^{\prime}, t, k}\right\rangle_{t}\right)^{2}\right\rangle_{t}}}\right\rangle k, x \neq x^{\prime}$.

Classical "noise correlations" are computed using trials as the internal variable, and averaged over pixel pairs

$\left\langle\frac{\left\langle b_{x, t, k} b_{x^{\prime}, t, k}\right\rangle_{k}}{\sqrt{\left\langle b_{x, t, k}^{2}\right\rangle_{k}} \sqrt{\left\langle b_{x^{\prime}, t, k}^{2}\right\rangle_{k}}}\right\rangle x \neq x^{\prime}$

they are a function of $t$, which again can be averaged over time.

As for above, if the process is stationary and the time window is long enough, both computations converge to the same value. And again, only our computation allows investigating correlations in specific frequency bands.

\section{Appendix B: The Observed Correlation Decrease Can be Explained by a Decrease in Correlated Activity Alone, Without any Increase in Uncorrelated Activity}

We report an important stimulus-induced decrease of the correlation between "noise signals" (i.e., after normalization by the average response) recorded from different cortical locations, with respect to the correlation in the absence of stimulation. Considering the signals as composed by a correlated and an uncorrelated component, this effect could result from a decrease of the correlated one, either alone-thus resulting in a comparatively large reduction in signal amplitude (measured as std.) or together with an increase of the uncorrelated one, which results in a smaller signal amplitude decrease. Below, we formally address the question of whether the size of the amplitude decrease in our data is large enough to be compatible with both possibilities or if it allows only for the second one.

For this purpose, we shall calculate the minimal size of the signal std. decrease needed to account for a given decrease in correlation, without any concomitant increase in uncorrelated activity. Let us consider two spontaneous signals $y_{1}$ and $y_{2}$ recorded at two different locations and express each of those as a sum

$y_{i}=x_{0}+x_{i}$ 
where $x_{0}$ is a signal common to $y_{1}$ and $y_{2}$, and $x_{1}$ and $x_{2}$ (and obviously $x_{0}$ ) are uncorrelated. For the sake of simplicity, we assume here that the scaling of $x_{0}$ is the same in both $y_{1}$ and $y_{2}$, and also that $x_{1}$ and $x_{2}$ have the same norm (i.e., std.), and introduce the ratio $s=\left\|x_{0}\right\|^{2} /\left\|x_{1}\right\|^{2}=\left\|x_{0}\right\|^{2} /\left\|x_{2}\right\|^{2}$, which expresses how much the common signal contributes to the total signal.

We also consider a second set of evoked "noise signals" $y_{1}^{\prime}$ and $y_{2}^{\prime}$, where the contribution of the common signal has been reduced by a factor $\alpha<1$, but the norm of the uncorrelated signals is unchanged

$y_{i}^{\prime}=\alpha x_{0}^{\prime}+x_{i}^{\prime}$,

with $\left\|x_{0}^{\prime}\right\|=\left\|x_{0}\right\|$ and $\left\|x_{i}^{\prime}\right\|=\left\|x_{i}\right\|$.

Below, we will compute the correlation decrease $\frac{c^{\prime}}{c} \frac{\operatorname{corr}\left(y_{1}^{\prime}, y_{2}^{\prime}\right)}{\operatorname{corr}\left(y_{1}, y_{2}\right)}$, as a function of $\alpha$, the original correlation $c=\operatorname{corr}\left(y_{1}, y_{2}\right)$ and of the decrease in std. $r=\frac{\left\|y_{i}^{\prime}\right\|}{\left\|y_{i}\right\|}$.

The correlation values are

$c=\frac{\left\langle y_{1} \cdot y_{2}\right\rangle}{\left\|y_{1}\right\| \cdot\left\|y_{2}\right\|}=\frac{\left\|x_{0}\right\|^{2}}{\left\|x_{0}\right\|^{2}+\left\|x_{i}\right\|^{2}}=\frac{s^{2}}{s^{2}+1}$,

and

$c^{\prime}=\frac{\alpha^{2} s^{2}}{\alpha^{2} s^{2}+1}$

From Eq. (3), we can express $s^{2}$ as a function of $c$

$s^{2}=\frac{c}{1-c}$.

The square of the total signal decrease $r$ is

$r^{2}=\frac{\left\|y_{i}^{\prime}\right\|^{2}}{\left\|y_{i}\right\|^{2}}=\frac{\alpha^{2}\left\|x_{0}\right\|^{2}+\left\|x_{i}\right\|^{2}}{\left\|x_{0}\right\|^{2}+\left\|x_{i}\right\|^{2}}=\frac{\alpha^{2} s^{2}+1}{s^{2}+1}$,

from which we can express $\alpha$ as a function of $r$ and $s$

$\alpha^{2}=\frac{r^{2} s^{2}+r^{2}-1}{s^{2}}$

Finally, we have:

$\frac{c^{\prime}}{c}=\frac{\alpha^{2}}{r^{2}}$, and using Eqs. (5) and (7),

$\frac{c^{\prime}}{c}=1-\frac{1-c}{c}\left(\frac{1}{r^{2}}-1\right)$.

If the decrease is small, $r$ is close to 1 , approximating at the first order: $\frac{1}{r^{2}}-1 \sim 2(1-r)$, then allows rewriting Eq. (8) as

$\frac{c^{\prime}-c}{c} \sim 2 \frac{1-c}{c}(r-1)=2 \frac{1-c}{c} \frac{\left\|y_{i}^{\prime}\right\|-\left\|y_{i}\right\|}{\left\|y_{i}\right\|}$.

For a correlation between spontaneous signals $c$ around 0.2 as in our data, the factor $2 \frac{1-c}{c}$ relating signal std. decrease and correlation decrease is as high as 8 .

As mentioned in the main text, our correlation decrease does not exceed the one predicted by Eq. (8): $\frac{\left\|y_{i}^{\prime}\right\|-\left\|y_{i}\right\|}{\left\|y_{i}\right\|}$ is on average equal to $-6.9 \%$ and $c$ to 0.194 . If these changes were solely due to the decrease of a common (i.e., fully correlated) signal, one would observe a correlation decrease $\frac{c^{\prime}-c}{c}$ of $-63.9 \%$ ! This is clearly more than the average correlation drop of $-37.4 \%$ observed in our data, which can, therefore, be explained by a simple decrease in correlated activity, without the need of invoking an increase in uncorrelated activity (although not ruling it out). It is also possible that a part of the uncorrelated component decreases as well, but necessarily to a much lesser extent than the correlated component (otherwise, the correlation between signals from different locations would simply remain constant).

\section{Disclosures}

The authors have no relevant financial interests in the paper and no other potential conflicts of interest to disclose.

\section{Acknowledgments}

The authors thank F. Barthelemy and M. Martin for help with the monkey training, and F. Chavane for fruitful discussions on the paper. This work was supported by the European Union (Integrated Project BrainScales), Award No. IST-FET-2010-269921; a French-Hungarian International ANR grant MULTISCALEFUNIM; and recurrent funding by CNRS and Aix-Marseille Université.

\section{References}

1. A. Arieli et al., "Coherent spatiotemporal patterns of ongoing activity revealed by real-time optical imaging coupled with single-unit recording in the cat visual cortex," J. Neurophysiol. 73(5), 2072-2093 (1995).

2. A. Arieli et al., "Dynamics of ongoing activity: explanation of the large variability in evoked cortical responses," Science 273(5283), 18681871 (1996).

3. J. Anderson et al., "Stimulus dependence of two-state fluctuations of membrane potential in cat visual cortex," Nat. Neurosci. 3(6), 617621 (2000).

4. Y. Shu et al., "Barrages of synaptic activity control the gain and sensitivity of cortical neurons," J. Neurosci. 23(32), 10388-10401 (2003).

5. R. Cossart, D. Aronov, and R. Yuste, "Attractor dynamics of network UP states in the neocortex," Nature 423(6937), 283-288 (2003).

6. J. Fiser, C. Chiu, and M. Weliky, "Small modulation of ongoing cortical dynamics by sensory input during natural vision," Nature 431(7008), 573-578 (2004).

7. T. Kenet et al., "Spontaneously emerging cortical representations of visual attributes," Nature 425(6961), 954-956 (2003).

8. P. Berkes et al., "Spontaneous cortical activity reveals hallmarks of an optimal internal model of the environment," Science 331(6013), 83-87 (2011).

9. C. Gómez-Laberge et al., "Bottom-up and top-down input augment the variability of cortical neurons," Neuron 91(3), 540-547 (2016).

10. M. H. Chang, K. M. Armstrong, and T. Moore, "Dissociation of response variability from firing rate effects in frontal eye field neurons during visual stimulation, working memory, and attention," J. Neurosci. 32(6), 2204-2216 (2012).

11. B. White, L. F. Abbott, and J. Fiser, "Suppression of cortical neural variability is stimulus- and state-dependent," J. Neurophysiol. 108(9), 2383-2392 (2012).

12. M. M. Churchland et al., "Stimulus onset quenches neural variability: a widespread cortical phenomenon," Nat. Neurosci. 13(3), 369-378 (2010).

13. I. M. Finn, N. J. Priebe, and D. Ferster, "The emergence of contrastinvariant orientation tuning in simple cells of cat visual cortex," Neuron 54(1), 137-152 (2007).

14. C. Monier et al., "Orientation and direction selectivity of synaptic inputs in visual cortical neurons: a diversity of combinations produces spike tuning," Neuron 37(4), 663-680 (2003).

15. B. J. He, "Spontaneous and task-evoked brain activity negatively interact," J. Neurosci. 33(11), 4672-4682 (2013).

16. G. Orbán et al., "Neural variability and sampling-based probabilistic representations in the visual cortex,"Neuron 92(2), 530-543 (2016). 
17. M. A. Smith and A. Kohn, "Spatial and temporal scales of neuronal correlation in primary visual cortex," J. Neurosci. 28(48), 12591 (2008).

18. M. W. Oram, "Visual stimulation decorrelates neuronal activity," J. Neurophysiol. 105(2), 942-957 (2011).

19. T. Deneux and A. Grinvald, "Milliseconds of sensory input abruptly modulate the dynamics of cortical states for seconds," Cereb. Cortex (2016).

20. S. Chemla and F. Chavane, "Voltage-sensitive dye imaging: technique review and models," J. Physiol. Paris 104(1-2), 40-50 (2010).

21. M. A. Smith and M. A. Sommer, "Spatial and temporal scales of neuronal correlation in visual area v4," J. Neurosci. 33(12), 5422-5432 (2013).

22. A. Arieli, A. Grinvald, and H. Slovin, "Dural substitute for long-term imaging of cortical activity in behaving monkeys and its clinical implications," J. Neurosci. Methods 114(2), 119-133 (2002).

23. E. Shtoyerman et al., "Long-term optical imaging and spectroscopy reveal mechanisms underlying the intrinsic signal and stability of cortical maps in V1 of behaving monkeys," J. Neurosci. 20(21), 8111-8121 (2000).

24. K. R. Gegenfurtner and M. J. Hawken, "Interaction of motion and color in the visual pathways," Trends Neurosci. 19(9), 394-401 (1996).

25. A. W. Roe et al., "Toward a unified theory of visual Area V4," Neuron 74(1), 12-29 (2012).

26. D. Shoham et al., "Imaging cortical dynamics at high spatial and temporal resolution with novel blue voltage-sensitive dyes," Neuron 24(4), 791-802 (1999).

27. S. Chemla and F. Chavane, "A biophysical cortical column model to study the multi-component origin of the VSDI signal," NeuroImage 53(2), 420-438 (2010).

28. L. B. Cohen et al., "Changes in axon fluorescence during activity: molecular probes of membrane potential," J. Membr. Biol. 19(1), 1-36 (1974).

29. B. M. Salzberg et al., "Optical recording of neuronal activity in an invertebrate central nervous system: simultaneous monitoring of several neurons," J. Neurophysiol. 40(6), 1281-1291 (1977).

30. G. Deco and E. Hugues, "Neural network mechanisms underlying stimulus driven variability reduction," PLoS Comput. Biol. 8(3), e1002395 (2012).

31. G. Deco and V. K. Jirsa, "Ongoing cortical activity at rest: criticality, multistability, and ghost attractors," J. Neurosci. 32(10), 3366-3375 (2012).

32. K. Rajan, L. F. Abbott, and H. Sompolinsky, "Stimulus-dependent suppression of chaos in recurrent neural networks," Phys. Rev. E 82(Pt. 1), 011903 (2010).

33. B. Blumenfeld, D. Bibitchkov, and M. Tsodyks, "Neural network model of the primary visual cortex: from functional architecture to lateral connectivity and back," J. Comput. Neurosci. 20(2), 219-241 (2006).

34. D. L. Ringach, "Spontaneous and driven cortical activity: implications for computation," Curr. Opin. Neurobiol. 19(4), 439-444 (2009).

35. J. Fiser et al., "Statistically optimal perception and learning: from behavior to neural representations," Trends Cognit. Sci. 14(3), 119-130 (2010).
36. M. V. Sanchez-Vives and M. Mattia, "Slow wave activity as the default mode of the cerebral cortex," Arch. Ital. Biol. 152(2-3), 147-155 (2014).

Thomas Deneux develops data analysis methods for high-throughput recordings of neural activity at UNIC, Centre National de la Recherche Scientifique (CNRS), France. During his $\mathrm{PhD}$, supervised by Olivier Faugeras at INRIA, Paris, France, he focused on human brain data analysis, whereas he studied spontaneous activity and multisensory integration using VSD and two-photon microscopy during his postdoctoral trainings, supervised by Amiram Grinvald at the Weizmann Institute, Rehovot, Israel; Ivo Vanzetta at Institut de Neurosciences de la Timone, Marseille, France; and Brice Bathellier at CNRS-UNIC, Paris, France.

Timothée Masquelier is a CNRS researcher in computational neuroscience. He uses numerical simulations and analytical calculations to gain understanding on how the brain works, and more specifically on how neurons process and transmit information through action potentials (a.k.a spikes), in particular in the visual modality. He is also interested in bioinspired computer vision and neuromorphic engineering. He was trained at Ecole Centrale Paris (Ingénieur 1999), MIT (MSc 2001), and Univ. Toulouse 3 ( $P h D 2008$, supervised by Simon Thorpe).

Maria A. Bermudez is a lecturer at the University of La Coruña, Spain, and her research focuses on the neurophysiology of the visual system. She received her PhD in neuroscience from the University of Santiago de Compostela, Spain. As a postdoctoral fellow, she first joined the laboratory of Professor Wolfram Schultz at Cambridge University, UK, to investigate reward related activity using single unit recordings and then joined the Institut de Neurosciences de la Timone in Marseille under the supervision of Ivo Vanzetta, to study vision in behaving monkeys using both optical imaging and psychophysics.

Guillaume S. Masson is a CNRS researcher in visual neurosciences. He investigates how the primate visual system processes visual motion information for both perception and eye movements, integrating behavioral, physiological, and modeling approaches. He has a background in neurosciences from Aix-Marseille University and was trained in neurophysiology with Professor Frederick A. Miles at the National Institutes of Health/National Eye Institute, Bestheda, Maryland, USA.

Gustavo Deco is an ICREA research professor and full professor at Universitat Pompeu Fabra, where he is also director of the Center of Brain and Cognition (CBC) and leads one of its groups (CNS). A world leader in computational neuroscience, Professor Deco's broad research interests include also neuropsychology, psycholinguistics, biological networks, statistical formulation of neural networks, and chaos theory.

Ivo Vanzetta is a CNRS researcher in photonic neuroimaging in vivo applied to neurosciences. He uses wide field optical imaging (intrinsic signals and voltage-sensitive dyes) and two-photon microscopy to investigate how the visual cortex of higher mammals processes sensory information, as well as to spatiotemporally characterize the hemodynamic responses elicited by cortical neuronal activity. 